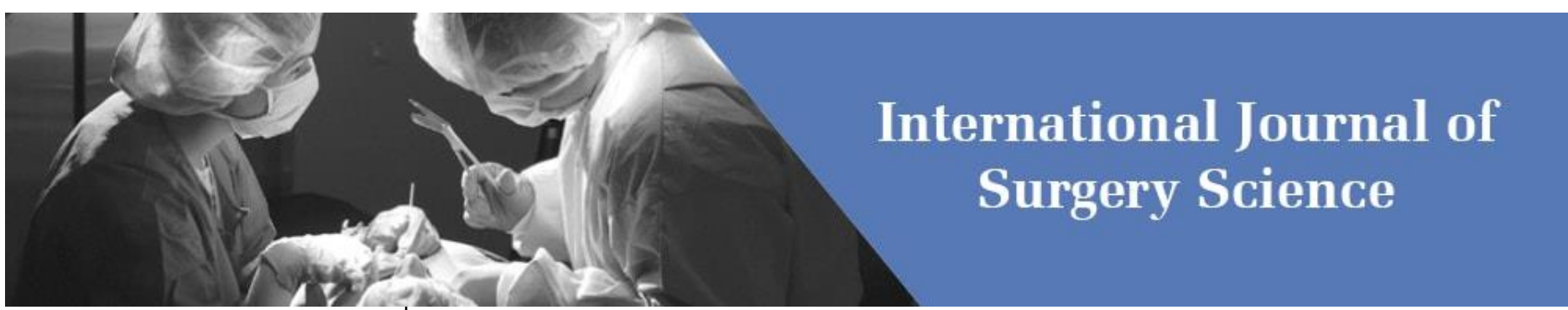

E-ISSN: 2616-3470

P-ISSN: 2616-3462

(C) Surgery Science

www.surgeryscience.com

2020; 4(1): 33-36

Received: 19-11-2019

Accepted: 23-12-2019

Dr. Anil Kumar MS

Professor, Department of General Surgery, JSS Medical College, JSS Academy of Higher Education and Research, Mysuru, Karnataka,

India

Dr. Surendra Mannan K MBBS, Junior Resident, Department of General Surgery, JSS Medical College, JSS Academy of Higher Education and Research, Mysuru, Karnataka, India
Corresponding Author: Dr. Surendra Mannan K MBBS, Junior Resident, Department of General Surgery, JSS Medical College, JSS Academy of Higher Education and Research, Mysuru, Karnataka, India

\section{Comparative study of early enteral feeding versus parenteral feeding in acute severe pancreatitis in JSS hospital}

\author{
Dr. Anil Kumar MS and Dr. Surendra Mannan K
}

DOI: https://doi.org/10.33545/surgery.2020.v4.i1a.307

\section{Abstract}

Background: Acute Severe acute pancreatitis is found in $20-30 \%$ of patients with pancreatitis and is associated with marked increased risk of complications, such as necrosis, abscess, formation of pancreatic pseudocyst and multiorgan failure, and also the disease manifests with a "systemic inflammatory response syndrome (SIRS) that culminates with hyper metabolism and high rate of protein catabolism; consequently, nutritional reserves are rapidly consumed and malnutrition may ensue unless a higher nutritional intake is provided, thus, early nutritional support plays a central role in the management of these patients. Parenteral nutritional support has long been the standard source of exogenous nutrients for these patients; however this is associated with many disadvantages, including dysfunction of the intestinal mucosal barrier, which, in turn, promotes sepsis of intestinal origin. Enteral nutrition, on the other hand, can maintain the integrity and function of the intestinal mucosal barrier, and thus the study intends to compare the efficacy of enteral versus parenteral feeding in influencing the outcomes in acute pancreatitis cases measured in terms of acute phase proteins, duration of ICU care, length of hospital stay.

Aim: To compare the efficacy of enteral feeds versus parenteral feeding in terms of, outcomes of acute pancreatitis patients, to assess acute phase response in both types of feeding and measuring the length of hospital stay including length of ICU stay.

Methods: Patients admitted with diagnosis of acute pancreatitis will be randomly grouped into group A and B by odd and even number hospital admission, Sample Size is considered as 100 with 50 in group A receiving enteral feeding and 50 in group $\mathrm{B}$ receiving parenteral feeding, considering the mean difference in hospital stay of 6 days and SD in each group as 12 days.

Results: It was seen that patients who received early enteral nutrition had better outcomes in terms of acute phase proteins, shorter length of hospital stay (p-value:0.038, t-test:7.868) and shorter length of ICU care (p-value: 0.044, t-test:1.094) that compared with total parenteral nutrition group.

Conclusion: It can be concluded that in our study, early enteral nutrition can be started safely and efficient when started "within 48 hours of admission" which had better outcomes in terms of acute phase proteins, shorter duration of ICU care and length of hospital stay and also cost effective that compared to those patients who received total parenteral nutrition where lesser patients showed better outcomes, had increased hospital stay and was costlier.

Keywords: Systemic inflammatory response syndrome (SIRS), Total parenteral nutrition, enteral nutrition

\section{Introduction}

The definition of acute pancreatitis is an acute condition presenting with abdominal pain and is associated with the raised pancreatic enzyme ( 3 fold) levels in the blood or urine as a result of pancreatic inflammation. It is associated with marked increased risk of complications, such as necrosis, abscess, formation of pancreatic pseudocyst and multiorgan failure". And also the disease manifests with a "systemic inflammatory response syndrome (SIRS) that culminates with hyper metabolism and high rate of protein catabolism; consequently, nutritional reserves are rapidly consumed and malnutrition may ensue unless a higher nutritional intake is provided, thus, early nutritional support plays a central role in the management of these patients.

Pancreatic rest by keeping the patient nil by mouth and nutritional support by parenteral feeding has long been the standard source of exogenous nutrients for these patients; however this is associated with many disadvantages, including dysfunction or atrophy of the intestinal mucosal gut barrier, which, in turn, promotes sepsis of intestinal origin ${ }^{[2]}$. Also, bypassing intestine as the prime route of nutrient administration contributes to the loss of "mucosal barrier's integrity, 
exacerbating the stress and Systemic inflammatory response aggravating the disease severity and potentially leading to the development of sepsis, nosocomial infections and multiple organ failure.

Enteral nutrition, on the other hand, can maintain the integrity and function of the intestinal mucosal barrier ${ }^{[3]}$, and thus this study intends to "compare the efficacy of enteral versus parenteral feeding with regards to influencing the outcomes in acute pancreatitis cases.

\section{Methods and Materials}

It is an Randomized control study conducted in JSS hospital, Patients admitted with diagnosis of acute pancreatitis will be randomly grouped into group A and B by odd and even number hospital admission after satisfying inclusion and exclusion criteria, Sample Size is considered as 100 with 50 patients in group A receiving enteral feeding (kitchen feeds) through nasogastric route, started within 48 hours of admission and 50 in group B receiving standard parenteral formula feeding through peripheral line, considering the mean difference in hospital stay of 6 days and SD in each group as 12 days.

\section{Inclusion and Exclusion criteria}

Patients with a serum amylase more than threefold and clinical evidence of acute pancreatitis will be enrolled. Patients with 3 or more Glasgow points indicating severe disease, etiological factors like Alcohol, gallstone, trauma, drug induced, age: 20-50 years and patients giving consent was included in the study, and patients with known chronic pancreatitis, presenting more than 48 hours after admission, patients not tolerating enteral feeding through nasogastric tube, immune-compromised patients, established septicemia, multi organ failure patients, associated malignancy of GB or pancreas were excluded from this study.

\section{Statistical analysis}

All data will be taken as mean, median, standard deviation, interquartile range, proportions and inferential statistics: chi square, independent T test.

All the measures will be done using SPS 21.0 software.

$\mathrm{P}<0.5$ will be considered insignificant.

\section{Results and Inference}

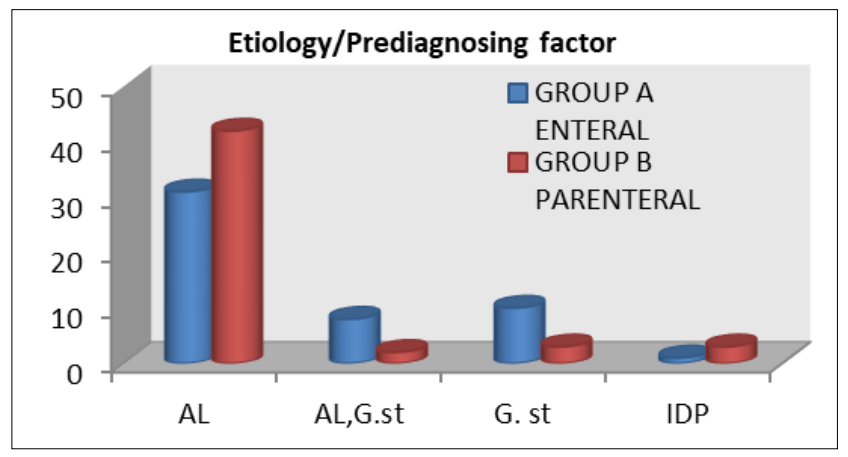

Fig 1: Shows distribution of etiological factors, Al-alcoholic; AL, G.st-alcohol with gallstone; G.st- gall stone; IDP-idiopathic.

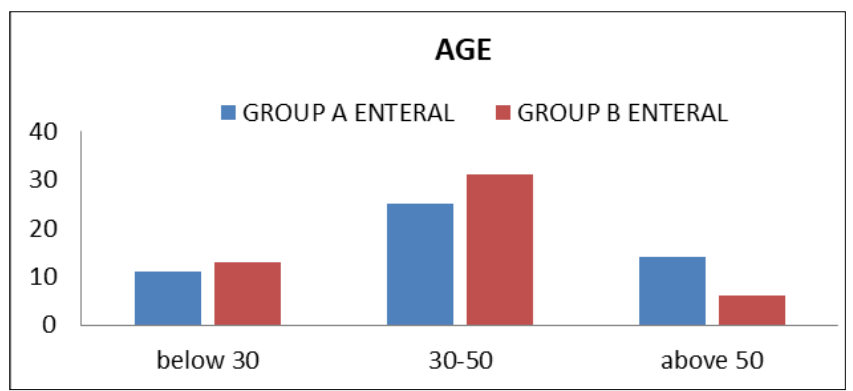

Fig 2: Shows age distribution, in our study majority of the patients come under average age of 30-50 years.

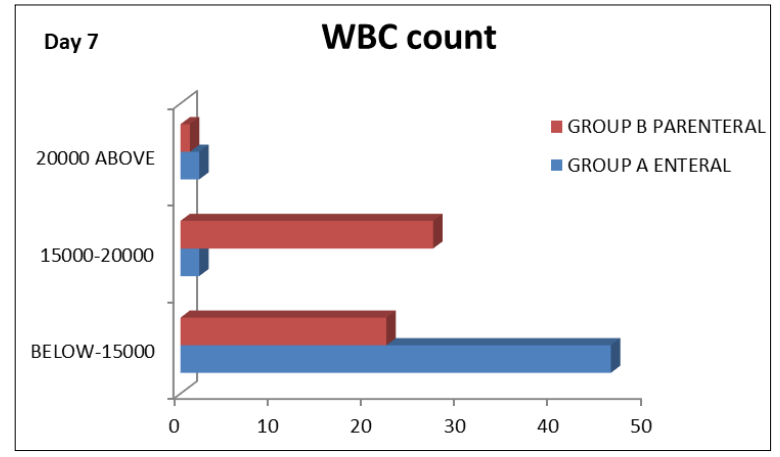

Fig 3: shows WBC counts repeated on day 7 after enteral and parenteral nutrition. It can be seen that out of 50 patients, maximum of $92 \%$ patients, had significant drop in the counts below $15 \times 10^{9} / 1$. and In group B those who received total parenteral nutrition, out of 50 patients, maximum of 54\% patients, still had elevated total leukocyte counts, above $15 \times 10^{9} / 1$.

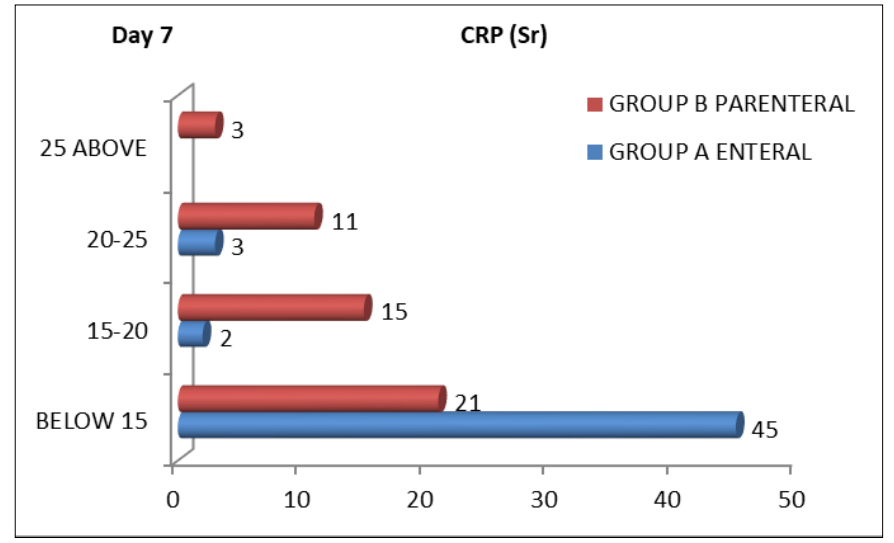

Fig 4: Shows the distribution of c- reactive protein, The drop in serum $\mathrm{C}$ - reactive protein was more pronounced in group A patients who received early $(<48 \mathrm{hrs})$ enteral nutrition. 


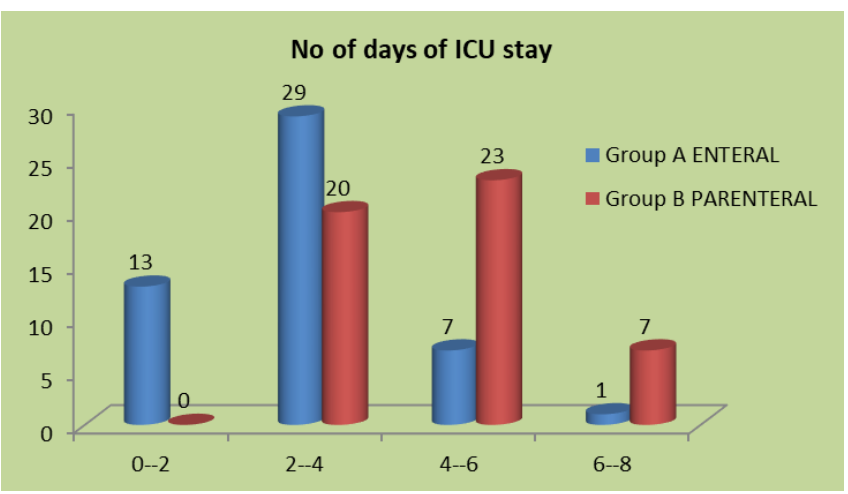

Fig 5: provides insight with regard to length of ICU care, group A patients who received early enteral feeding had shorter stay in ICU that compared with group B patients receiving parenteral nutrition.

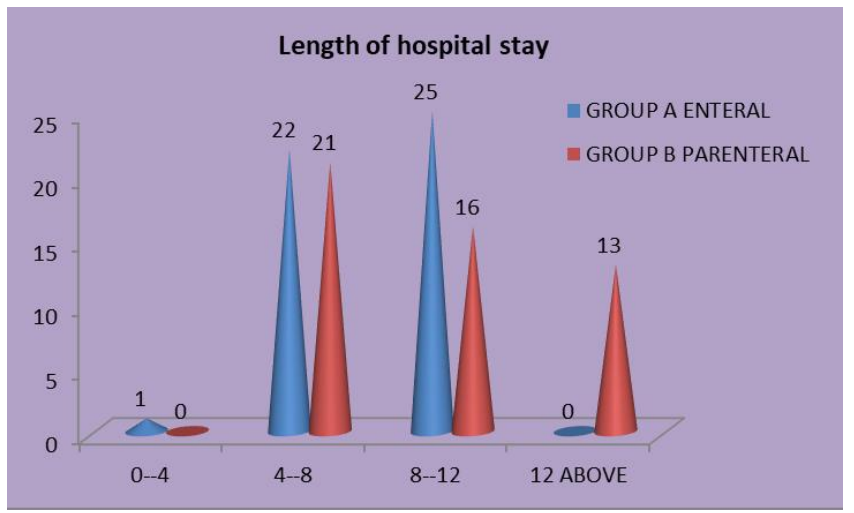

Fig 6: Shows distribution of patient's total length of hospital stay, in group A, maximum of $50 \%$ was admitted for a period of 8-12 days and 22 patients stayed for 4 to 8 days. In Group B patients, 21 of them was admitted for 4 to 8 days, 16 patients were in hospital for 8 to 12 days and 13 patients had a longer duration of stay of more than 12 days. Therefore, the early enteral nutrition patients had shorter hospital stay that compared with patients who received total parenteral nutrition.

\section{Discussion}

Early enteral feeding in cases of acute severe pancreatitis is advised in most current ICU guidelines ${ }^{[4]}$. In our study, a total of 100 patients diagnosed with acute severe pancreatitis after respective investigational parameters were grouped into 1:1 after satisfying inclusion and exclusion criteria's, i.e., group A (50 patients) receiving enteral nutrition started within 48 hours of admission and group B (50 patients) receiving total parenteral nutrition which followed 'pancreatic rest' theory". Male gender was commonly encountered and maximum of $50 \%$ and $62 \%$ of patients in group A and group B patients had the mean age of 42 years.

In the distribution of Etiology/Prediagnosing factor of patients, Out of 50 patients, maximum i.e. $62 \%$ and $82 \%$ had $\mathrm{AL}$ (alcohol) as etiology in group A and group B respectively, making AL (alcohol) being most common cause ${ }^{[5]}$. Patients underwent initial investigations, the distribution of Serum Amylase of patients, out of 50 patients, maximum 34\% of them fall in range 1500-2000 in group A enteral and maximum 38\% of below 600 range in group B parenteral. Similarly, the distribution of serum Lipase of patients, out of 50, maximum $54 \%$ of them fall in ranges above 2500 and maximum of $46 \%$ falling in ranges 1500-2000, in Group A and Group B respectively.

Acute phase proteins were assessed in terms of WBC count, serum c-reactive proteins. In the distribution of wbc counts of patients, out of 50 , maximum $66 \%$ and $40 \%$ of them were in the range $15 \times 10^{9} / \mathrm{L}$ to $20 \times 10^{9} / \mathrm{L}$ in Group A and Group B respectively, Hence, most patients presented with a wbc count more than $15 \times 10^{9} / \mathrm{L}$ and it was statistically significant with $\mathrm{p}$ (0.005).

The distribution of serum c-reactive protein, out of 50 , maximum $36 \%$ and $38 \%$ of them in the range above 15 units in both Group A and Group B respectively with statistical significance of $\mathrm{p}(0.038)$.

Hence there was no difference between two groups in terms of demographics and acute phase proteins on initial presentation.

In our study, with due consent of the patients, enteral nutrition was started within 48 hours of admission. Prepyloric nasogastric delivery after gastric emptying, intermittent feeds was administered, with choice of feed being Polymeric feeds, commercial type; contains nitrogen as whole protein, carbohydrate as partially hydrolyzed starch, and fat as long chain triglycerides derived from safflower oil or soybean oil or mixture of long chain and medium chain triglycerides derived from coconut oil, product name Ensure, Fresubin in 50 patients randomized to Group A.

TPN(Standard commercial solutions) started with peripheral line route in 50 patients in Group B and acute phase proteins was assessed with WBC count and serum C-Reactive protein on Day 5 and Day 7. Out of 50, maximum $92 \%$ of patients had their 'wbc' counts below $15 \times 10^{9} / \mathrm{L}$ in group A enteral nutrition compared to group B parenteral who's ranges was in $15 \times 10^{9} / \mathrm{L}-$ $20 \times 10^{9} / \mathrm{L}$ and serum $\mathrm{c}$ - reactive protein, out of 50 patients, maximum $90 \%$ and $42 \%$ of them in group A and group B were below $15 \mathrm{mg} / \mathrm{L}$ on day 7 .

Similarly APACHE II score were compared, which fell significantly in enteral group of patients, post nutritional, as seen by maximum of $76 \%$ of patients having below 8 scores that compared to parenteral group who were only $30 \%$. It can be seen that there is significant decrease in WBC count and serum $\mathrm{C}$ - reactive protein in patients started with enteral nutrition within 48 hours as compared to patients who received total parenteral nutrition. These finding seem to co relate with the study conducted by Peterson et al. ${ }^{[6]}$ and Kudsk et al. ${ }^{[7]}$ in their series of trauma patients suggesting that enteral feeding modulates gut pathophysiology and decreases systemic inflammatory response. A J C Windsor et al also concluded that compared with parenteral feeding, enteral nutrition attenuates the acute phase response and improves disease severity in acute pancreatitis patients.

Patients ICU care and hospital stay was compared similarly and out of 50 patients in group A enteral, maximum of $58 \%$ of patients were in ICU for a period of 2-4 days and maximum of $50 \%$ of patients total length of hospital stay was 8-12 days with mean duration of 3 and 10 days. On the other hand, out of 50 patients in group B parenteral, maximum of $46 \%$ of patients were in ICU for 4-6 days and maximum of $42 \%$ of patient's total length of hospital stay was 8-12 days with mean duration of 5 and 10 days, there is statistical significance of $p(0.044)$ and $p$ $(0.039)$ respectively. There was 1 mortality in enteral group and 2 mortality in parenteral group, concluding length of ICU and hospital stay was comparatively less in group A receiving enteral nutrition "within 48 hours of admission" and that compared with group B receiving parenteral nutrition. This was further supported by study done by Sun et al ${ }^{[8]}$. where there was significant decrease in incidences of systemic inflammatory response, multiple organ failure, length of ICU care and length of hospital stay, however it did not compare the differences in mortality. 


\section{Conclusion}

In patients with predicted severe pancreatitis, nutritional support should be the most important primary therapy and may begin within 48 hours of admission. It can be concluded that in our study, early enteral nutrition can be started safely and efficient when started within 48 hours of admission, which had better outcomes in terms of acute phase proteins, shorter duration of ICU care, length of hospital stay and also cost effective, that compared to those patients who received total parenteral nutrition where lesser patients showed better outcomes, had increased hospital stay and was costlier. International published guidelines recommend that either elemental or polymeric enteral nutrition formulations can be used in acute pancreatitis ${ }^{[9]}$.

\section{Conflicts}

None

\section{References}

1. Vieira JP, Araújo GF, Azevedo JRA, Goldenberg A, Linhares MM. Parenteral nutrition versus enteral nutrition in severe acute pancreatitis. Acta Cir Bras. 2010; 25(5)3094.

2. Wang G, Wen J, Xu L, Zhou S, Gong M, Wen P, et al. Effect of enteral nutrition and ecoimmunonutrition on bacterial translocation and cytokine production in patients with severe acute pancreatitis. J Surg Res. 2013; 183:592-7.

3. Heyland DK, Dhaliwal R, Drover JW, Gramlich L, Dodek P. Canadian clinical practice guidelines for nutrition support in mechanically ventilated, critically ill adult patients. J Parenter Enteral Nutr. 2003; 27:355-73.

4. Mirtallo JM, Forbes A, McClave SA, Jensen GL, Waitzberg DL, Davies AR. International consensus guidelines for nutrition therapy in pancreatitis. JPEN J Parenter Enteral Nutr. 2012; 36:284-291.

5. Gloor B, Müller CA, Worni M, et al. Late mortality in patients with severe acute pancreatitis. Br J Surg. 2001; 88:975-9.

6. Peterson VM, Moore EE, Jones TN, et al. Total enteral nutrition versus total parenteral nutrition after major torso injury: attenuation of hepatic protein reprioritization. Surgery. 1988; 104:199-207.

7. Kudsk KA, Minard G, Wojtysiac SL, et al. Visceral protein response to enteral versus parenteral nutrition and in patients with trauma. Surgery. 1994; 116:516-23.

8. Sun JK, Mu XW, Li WQ, Tong ZH, Li J, Zheng SY. Effects of early enteral nutrition on immune function of severe acute pancreatitis patients. World J Gastroenterol. 2013; 19:917-22.

9. Working Group IAP/APA Acute Pancreatitis Guidelines. IAP/APA evidence-based guidelines for the management of acute pancreatitis. Pancreatology. 2013; 13(4 Suppl 2):e1e15. PMID: 24054878. 